\title{
Creativity and Blocking: No Evidence for an Association
}

\author{
Tara Zaksaite $^{1}$ (D) , Peter M. Jones ${ }^{2}$ (D) , and Chris J. Mitchell² \\ 1 The Open University, UK \\ 2 Plymouth University, UK \\ ${ }^{*}$ corresponding author gintare.zaksaite@open.ac.uk
}

Received 14 April 2017; accepted 26 September 2017; published 21 November 2017.

\begin{abstract}
Creativity is an important quality that has been linked with problem solving, achievement, and scientific advancement. It has previously been proposed that creative individuals pay greater attention to and are able to utilize information that others may consider irrelevant, in order to generate creative ideas (e.g., Eysenck, 1995). In this study we investigated whether there was a relationship between creativity and greater learning about irrelevant information. To answer this question, we used a self-report measure of creative ideation and a blocking task, which involved learning about irrelevant stimuli. We failed to find evidence for this association, with a Bayes Factor indicating support for no relationship between these measures. While it is possible that a different measure of creative ideation, for example one which does not rely on self-report, may produce different results, a more lucrative research direction may be focusing on the link between creativity and cognitive flexibility, in line with suggestions by Zabelina and Robinson (2010).
\end{abstract}

Keywords: blocking; creative ideation; creativity; learning; Runco Ideational Behavior Scale; blocking.

\section{Creativity and Blocking: No Evidence for an Association}

Creativity is an important quality which has value in problem solving (Sawyer, 2012), education (Lewis, 2009), and has been related to advancements in engineering, mathematics, and technology (e.g., Plucker, Beghetto, \& Dow, 2004). Thus, it is important to study creativity in order to identify people, properties and circumstances 
associated with it, to investigate the possibility of maximizing related positive outcomes and recognize any associated risks. It has been previously suggested that creative people pay greater attention to irrelevant information (e.g., Eysenck, 1995; Kasof, 1997; Mendelsohn \& Griswold, 1966). Because attention guides learning (Mackintosh, 1975), this should result in creative people learning more about irrelevant information. This manuscript investigated whether a measure of creativity was related to greater learning about irrelevant stimuli within a causal learning task.

Causal learning describes a process of acquiring associations between causes and outcomes, allowing people to react appropriately to changes in their environment, predict events, and conserve resources. For example, a causal association may be learned between eating a food and getting a stomach ache, or between pressing a button and sound coming from a radio. A key question within associative learning is what determines the variability in the acquisition of such associations. Influential theories of associative learning have incorporated attention (e.g., Mackintosh, 1975; Pearce \& Hall, 1980). In particular, learning and attention have been shown to be greater for cues which predict their consequences very well, compared with cues which predict their consequences poorly (e.g., Le Pelley \& McLaren, 2001, 2003, 2004; Le Pelley, Mitchell, Beesley, George, \& Wills, 2016; Le Pelley, Vadillo, \& Luque, 2013; Lochmann \& Wills, 2003).

Attentional processes have also been implicated in creativity. It has been hypothesized that individuals high in creativity will exhibit defocused attention, also referred to as an "over-inclusive thinking style"; they will be more able to connect distant ideas and concepts and utilize information that others might consider irrelevant. For example, Dykes and McGhie (1976) found that participants high in creativity were better at remembering auditory stimuli that were instructed as irrelevant. This defocused attention is proposed to facilitate the idea-generation process of creativity (e.g., Ansburg \& Hill, 2003; Eysenck, 1995; Kasof, 1997; Mendelsohn \& Griswold, 1966).

Furthermore, creativity and performance in causal learning tasks have been linked. It has been found that individuals scoring high on creativity measures will exhibit greater learning about irrelevant stimuli than individuals scoring low in creativity. This has been evidenced by performance on latent inhibition tasks, involving learning about irrelevant stimuli (e.g., Carson, Peterson, \& Higgins, 2003; Kéri, 2011; Meyersburg, Carson, Mathis, \& McNally, 2014; Peterson, Smith, \& Carson, 2002). In a latent inhibition task, participants experience a repeated presentation of a stimulus (e.g., an image of a food) by itself. When this stimulus is later paired with an outcome (e.g., an allergic reaction), learning for this association is slower than for an association involving a novel stimulus. This is referred to as a latent inhibition effect (Lubow \& Moore, 1959). Latent inhibition effect has been found to be smaller in individuals who score high on creativity measures than those who score low, thus supporting the assertion that individuals high in creativity will pay more attention 
to irrelevant stimuli. In other words, individuals who are highly creative learn to treat irrelevant information as more relevant, than individuals who are less creative. Given the attentional links with both causal learning and creativity, differences in attention may be one reason for these findings.

While traditionally, the latent inhibition effect has been thought to be due to attention (e.g., Lubow, Alek, \& Arzy, 1975; Lubow, Schnur, \& Rifkin, 1976), alternative accounts have been proposed (e.g., Holmes \& Harris, 2010). In order to investigate whether an attentional link between causal learning and creativity exists, in this experiment we used a different type of learning effect related to irrelevant cues, known as blocking (Dickinson, Shanks, \& Evenden, 1984; Kamin, 1969). Blocking was selected due to its more established links with attention (e.g., Wills, Lavric, Croft, \& Hodgson, 2007). An example of a blocked stimulus is stimulus $\mathrm{B}$ on $\mathrm{A}+$ and $\mathrm{AB}+$ trials, on which stimulus A predicts the outcome, and A and B together predict the same outcome. For example, participants may be told that a fictional patient gets a food poisoning after eating apples $(A+)$, and they get a food poisoning after eating apples and bananas together $(\mathrm{AB}+)$. Stimulus $\mathrm{B}$ is redundant, because its companion, stimulus $\mathrm{A}$, predicts the outcome perfectly on A+ trials. Dickinson et al. (1984) found that B was thought to have caused the outcome to a lesser extent in a group which received $A+/ A B+$ training than in a group for which $\mathrm{A}+$ trials were omitted $(\mathrm{AB}+)$. In the same group of participants, the finding that $\mathrm{B}$ is thought to cause the outcome to a lesser extent than $\mathrm{D}$ on $\mathrm{A}+/ \mathrm{AB}+/ \mathrm{CD}+$ trials is referred to as the blocking effect.

Given the earlier proposed link between higher creativity and greater learning about irrelevant cues, we may expect that participants high in creativity would produce a smaller blocking effect. In order to measure creativity, we chose a measure relating to creative ideation, or ability to generate creative ideas. Because defocused attention has theoretically been proposed to enable individuals to generate creative ideas (e.g., Ansburg \& Hill, 2003; Eysenck, 1995; Kasof, 1997; Mendelsohn \& Griswold, 1966), such a measure should be best placed to detect a relationship between learning for irrelevant cues and creative-idea generation.

This is what we aimed to investigate in this experiment. Participants were asked to complete a learning task which included trial types necessary for blocking (Table 1). We also asked participants to complete a questionnaire of creative ideation, the Runco Ideational Behavior Scale (RIBS-S; Runco et al., 2014). This measure was chosen due to its focus on the process of generating novel ideas. We investigated whether there was a relationship between the size of the blocking effect displayed by the participants and their RIBS-S score. 
Table 1. The design of the learning task. The letters A-J represent different stimuli; "+" represents food poisoning and "-" an absence of food poisoning.

\begin{tabular}{lll}
\hline Stage 1 & Stage 2 & Test \\
\hline $\mathrm{A}+$ & $\mathrm{AB}+$ & $\mathrm{A}, \mathrm{B}$ \\
& $\mathrm{CD}+$ & $\mathrm{C}, \mathrm{D}$ \\
$\mathrm{E}-$ & $\mathrm{EF}+$ & $\mathrm{E}, \mathrm{F}$ \\
$\mathrm{GH}+$ & $\mathrm{K}+$ & $\mathrm{K}$ \\
& $\mathrm{L}-$ & $\mathrm{L}$ \\
$\mathrm{IJ}-$ & $\mathrm{IJ}-$ & $\mathrm{I}, \mathrm{J}$ \\
\hline
\end{tabular}

\section{Method}

\section{Participants}

126 participants in total assisted with this study (110 were female). They were aged 18-42 $(M=20.46, S D=3.86)$. They took part as a course requirement at Plymouth University. The study was described as an investigation into learning and personality. Inclusion criteria required participants to be over 18 years old, fluent in English, and have normal or corrected-to-normal vision and color vision.

\section{Materials}

The experiment was presented on a 22-inch desktop computer screen with a 1920 x 1080 resolution. The blocking task was designed, presented, and responses were recorded, using E-prime 2.0 software (Psychology Software Tools, PA, US). The questionnaire was designed and the associated data collected using Survey Monkey survey engine.

Blocking task. The foods used in the blocking task were asparagus, aubergine, avocado, carrots, cauliflower, lentils, mushroom, onion, pear, potato, pumpkin, and watermelon, which were randomly assigned to cues A-J for each participant. The outcomes were food poisoning and no food poisoning. 
Runco Ideational Behavior Scale: Short form (RIBS-S; Runco et al., 2014). This measure contained 19 statements, which relate to specific examples of everyday creative ideation (e.g., "I have ideas for arranging or rearranging the furniture at home"). Participants were asked to indicate the extent to which these statements applied to them on a 0 (Never) to 4 (Daily) response scale, with a total possible score of 76.

\section{Procedure}

The order of completion for the RIBS-S and the learning task was counterbalanced, with half of the participants completing the learning task followed by the RIBS-S measure and the other half vice versa.

The blocking task was self-paced. An allergist scenario was used for the blocking task, commonly used in associative learning experiments (e.g., Aitken, Larkin \& Dickinson, 2000). Participants were asked to imagine that they were diagnosing allergic reactions in a fictional patient. They were asked to observe which foods the patient ate and whether they experienced an allergic reaction or not. Images of foods sized $300 \times 300$ with text captions above were presented on the screen with black background, below the text "Patient eats the following food(s)": When a trial consisted of one image, this was presented at the center of the screen, while on trials with two images, one was presented on the left and one on the right. The outcomes were food poisoning, signified by text "The patient has food poisoning" and a sad face, and no food poisoning, signified by text "The patient has no food poisoning" and a smiley face. On each trial, participants were asked to rate the stimuli for their likelihood of food poisoning, on a scale of $1-9$ ( 1 = safe; 5 = uncertain; $9=$ dangerous). The scale was presented below the images, at the bottom of the screen and participants responded by clicking the appropriate number with a mouse. Immediate feedback followed with the appropriate outcome. The feedback was displayed on the screen for $3000 \mathrm{~ms}$. The progression to the next trial was immediate. Stage 1 consisted of 10 blocks of 4 trial types (A+, E-, GH+, IJ-), and Stage 2 of 5 blocks of 6 trial types ( $\mathrm{AB}+, \mathrm{CD}+, \mathrm{EF}+, \mathrm{IJ}-, \mathrm{K}+, \mathrm{L}-)$. The transition from Stage 1 to Stage 2 was seamless. At test, participants were asked to rate individual foods presented in Stage 2 on the same 9-point scale but received no feedback; each food was rated twice at test. 


\section{Results}

\section{Learning}

Responses in Stage 1, averaged across participants, are presented in Figure 1, and responses in Stage 2 are presented in Figure 2. These figures illustrate that participants were able to learn the pairings.

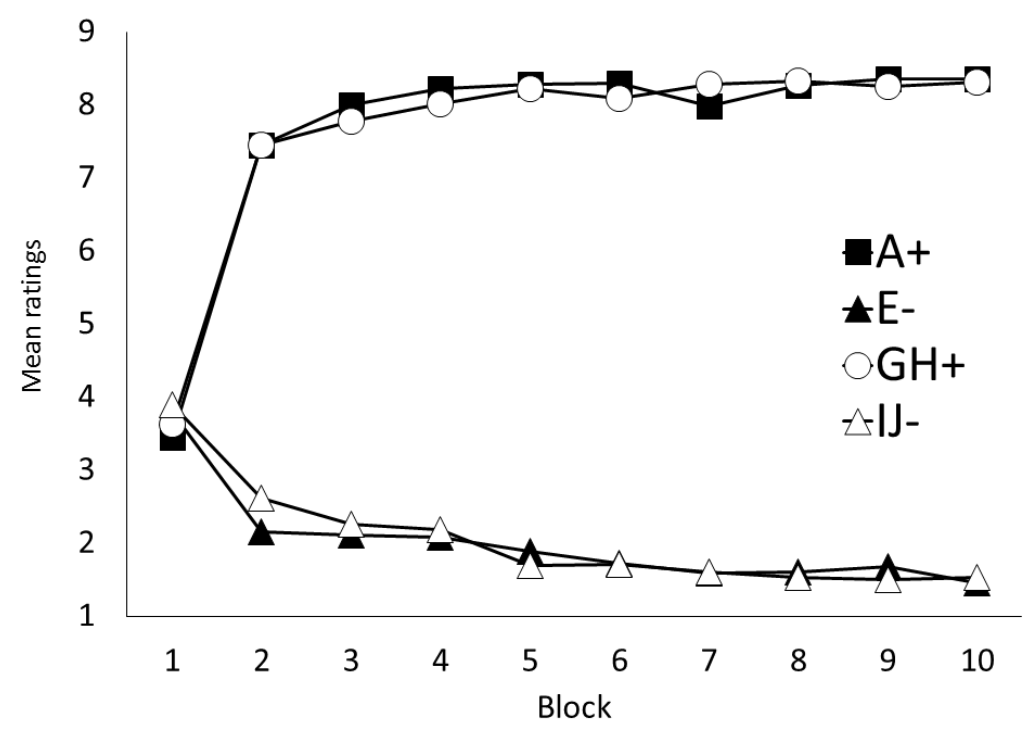

Figure 1. Responses in Stage 1.

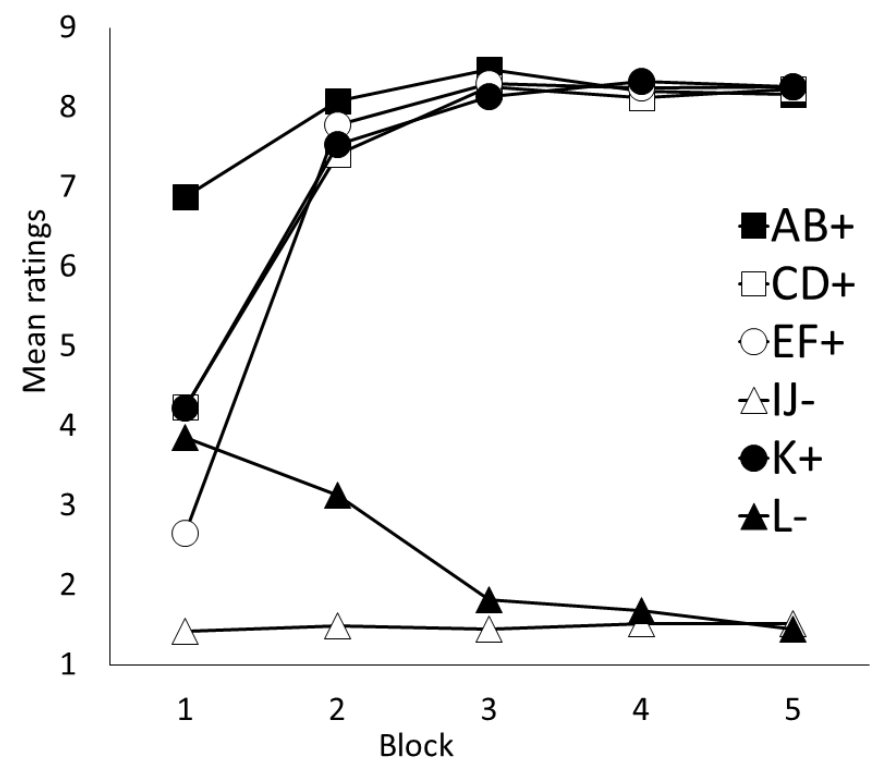

Figure 2. Responses in Stage 2. 


\section{Test}

Ratings for each cue at test are shown in Figure 3. A one-way ANOVA revealed a significant effect of cue, $F(3.13,391.33)=471.09, p<.001, \eta^{2}=.79$. Ratings for $\mathrm{C} / \mathrm{D}$ were greater than ratings for $\mathrm{B}$, indicating that blocking was observed, $t(125)=4.41, p<.001$.

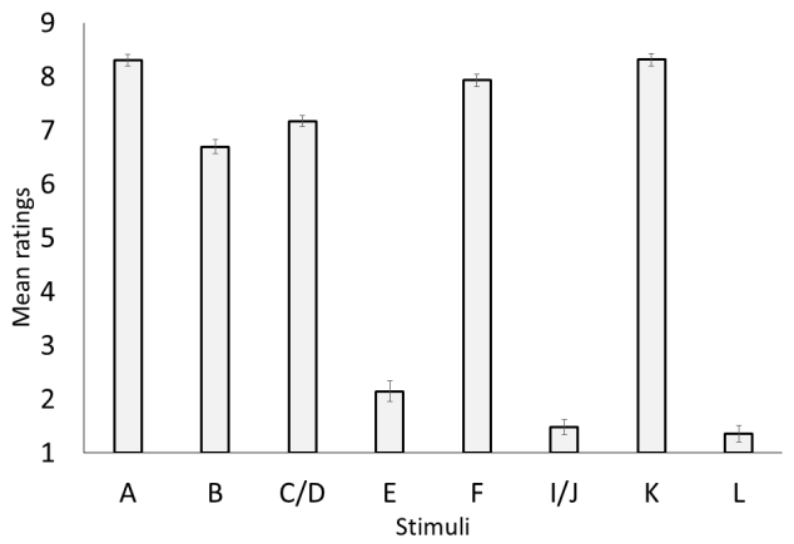

Figure 3. Mean causal ratings at test for each stimulus. Ratings for $C$ and $D$, and $I$ and $J$ were collapsed because these cues were treated equivalently.

Next we calculated a blocking-magnitude score for each participant by taking away ratings for B from ratings for C/D. In order to investigate whether there was a relationship between blocking and RIBS-S scores, a Pearson correlation coefficient was computed. This was not significant, $r(126)=-.022, p=.809, B F_{01}=8.72$ (Figure 4), indicating that there was no relationship between the magnitude of blocking and RIBS-S scores.

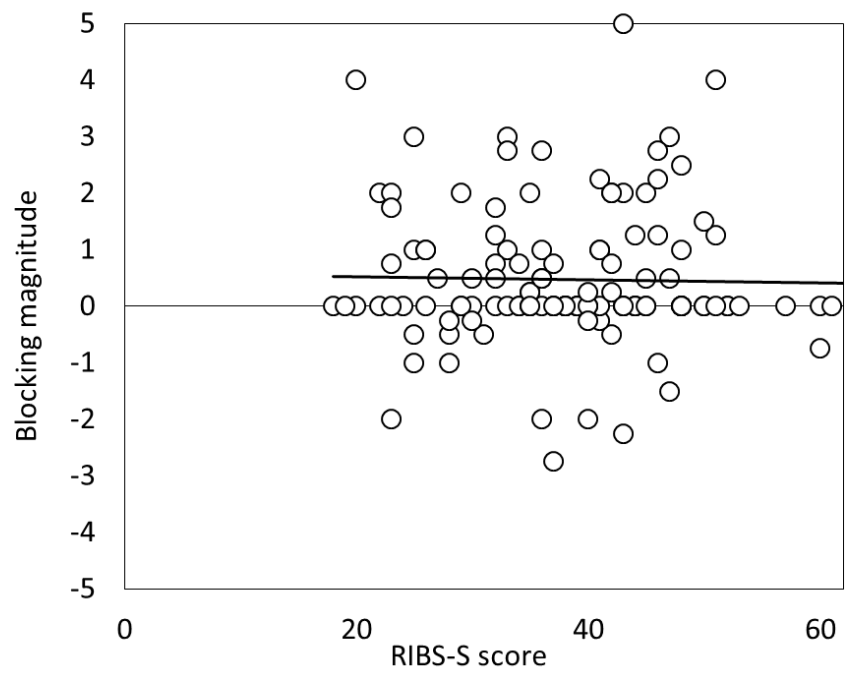

Figure 4. Correlation between blocking magnitude (ratings for $\mathrm{C}$ and $\mathrm{D}$ - ratings for $\mathrm{B}$ ) and RIBS-S score. 


\section{Discussion}

In this experiment we set out to investigate whether the magnitude of blocking, an effect related to learning about irrelevant cues, was related to creative ideation. It has previously been proposed that creative people pay more attention to and are able to utilize irrelevant information in order to generate creative ideas (e.g., Ansburg \& Hill, 2003; Eysenck, 1995; Mendelsohn \& Griswold, 1966), exhibiting a trait referred to as defocused attention. However, we did not find evidence to suggest that participants' scores on a measure of creative ideation were related to how much they learned about the blocked cue; the correlation between RIBS-S and blocking magnitude was near zero, with the Bayes Factor indicating support for the null hypothesis.

Overall, our findings did not support a link between greater learning about irrelevant information and ability to generate creative ideas. If the link between defocused attention and creativity exists, a relationship between these measures would have been expected. However, we do acknowledge the possibility that a different type of measure for coming up with creative ideas, perhaps one that does not rely on selfreport, e.g., divergent thinking, may produce different results; others may wish to investigate this further. However, it is worth noting that Runco et al. (2014) reported a strong positive relationship between participants' scores on RIBS-S and performance on divergent thinking tasks.

If no relationship between these measures exists, then how may these findings be reconciled with previous results? After all, several previous studies have indicated relationship between reduced latent inhibition and creativity (e.g., Carson et al., 2003; Kéri, 2011; Meyersburg et al., 2014; Peterson et al., 2002). One reason for the differential results may be differences between blocking and latent inhibition tasks. For example, blocking is usually estimated once learning for the cues is complete; participants are asked to provide ratings for how predictive a blocked versus a control stimulus is (e.g., Aitken, Larkin, \& Dickinson, 2000). On the other hand, latent inhibition often involves time taken to detect a predictive relationship between an incidental stimulus (e.g., the background color of the screen) and an element of the task. It is possible that creativity may be related to faster detection of a causal relationship rather than to attributing greater causality to irrelevant information.

Another difference between the previous studies and the current one is that they focused on creative achievement and personality whereas the present study focused on creative ideation. Arguably, creative achievement is the end result of creative ideation. Therefore it is possible that greater learning about irrelevant cues may be related to a different stage of creativity and not generating creative ideas specifically. In this case, defocused attention may still be related to creativity, but may only be exhibited by creative individuals under particular circumstances. In other words, there will be times when creative people can exhibit defocused attention, but this will not necessarily be stable throughout time. For example, this may only occur when people are 
directly involved in a creative activity, e.g., writing a piece of music. In relation to this, Zabelina and Robinson (2010) proposed that creative people, rather than exhibiting defocused attention, are able to utilize both defocused and focused attention, and switch between these two modes of attention as necessary. They referred to this ability as flexible cognitive control. In their study, they found that participants who scored highly on measures of creativity exhibited fewer reaction-time costs in a Stroop task on a trial-by-trial basis. A link between creativity and cognitive flexibility has also been reported by others (e.g., Chen et al., 2014; de Dreu, Nijstad, \& Baas, 2011). Given this link, it may be expected that creative participants will outperform others in other associative learning tasks which involve attentional switching. One example of this is intra-dimensional/extra-dimensional (ID/ED) shift (George \& Pearce, 1999; Mackintosh \& Little, 1969; Shepp \& Eimas, 1964). In such a task, participants are presented with stimuli which vary on several dimensions, for example: color and shape. They are asked to sort these stimuli into categories and receive corrective feedback. Over time, they learn that one particular dimension, e.g., color, is relevant and determines the categories. In the next part of the experiment, participants are presented with a different set of stimuli. In this part, for some stimuli, the dimension which determines the categories changes, and a previously irrelevant dimension determines the category, e.g., shape. It has been found that participants incur switching costs, taking longer to learn to categorize stimuli correctly when they are classified based on the previously irrelevant, versus the previously relevant, dimension. If Zabelina and Robinson's suggestions are correct, then participants who are more creative should exhibit lower switching costs than those who are less creative.

However, it is worth noting that creative processes may be the result of a different combination of focused and defocused attention than proposed by Zabelina and Robinson (2010), such that instead of creative participants exhibiting one extreme at any one time, they may utilize both at the same time. For a discussion of this alternative view of cognitive flexibility, please see Ionescu (2012).

To conclude, this experiment investigated whether there was a link between a measure of creative ideation, RIBS-S, and learning for irrelevant cues using a blocking paradigm. We failed to demonstrate this link. Given the theoretical link proposed between defocused attention and creativity (e.g., Eysenck, 1995; Kasof, 1997; Mendelsohn \& Griswold, 1966), this link would have been expected. Further studies may wish to investigate whether blocking is related to divergent thinking, and whether creativity may be related to switching performance on an ID/ED task.

\section{Acknowledgements}

The authors would like to thank Thea Ionescu and Mihaela Taranu for their reviews of this article. 


\section{References}

Aitken, M. R. F., Larkin, M. J. W., \& Dickinson, A. (2000). Super-learning of causal judgements. The Quarterly Journal of Experimental Psychology Section B, Comparative and Physiological Psychology, 53(1), 59-81.

Ansburg, P. I., \& Hill, K. (2003). Creative and analytic thinkers differ in their use of attentional resources. Personality and Individual Differences, 34(7), 1141-1152. doi:10.1016/S01918869(02)00104-6

Carson, S. H., Peterson, J. B., \& Higgins, D. M. (2003). Decreased latent inhibition is associated with increased creative achievement in high-functioning individuals. Journal of Personality and Social Psychology, 85(3), 499-506. doi:10.1037/0022-3514.85.3.499

Chen, Q., Yang, W., Li, W., Wei, D., Li, H., Lei, Q., Zhang, Q., \& Qiu, J. (2014). Association of creative achievement with cognitive flexibility by a combined voxel-based morphometry and resting-state functional connectivity study. NeuroImage, 102, 474-483.

de Dreu, C. K. WD., Nijstad, B. A. and Baas, M. (2011). Behavioral activation links to creativity because of increased cognitive flexibility. Social Psychological and Personality Science, 2(1), 72-80. doi:10.1177/1948550610381789

Dickinson, A., Shanks, D., \& Evenden, J. (1984). Judgement of act-outcome contingency: The role of selective attribution. Quarterly Journal of Experimental Psychology Section A: Human Experimental Psychology 36(1), 29-50. doi:10.1080/14640748408401502

Dykes, M., \& McGhie, A. (1976). A comparative study of attentional strategies of schizophrenic and highly creative normal subjects. British Journal of Psychiatry, 128(1), 50-56. doi:10.1192/bjp.128.1.50

Eysenck, H. J. (1995). Genius: The natural history of creativity. Cambridge, UK: Cambridge University Press.

George, D. N., \& Pearce, J. M. (1999). Acquired distinctiveness is controlled by stimulus relevance not correlation with reward. Journal of Experimental Psychology: Animal Behavior Processes, 25(3), 363-373. doi:10.1037/0097-7403.25.3.363

Holmes, N. M., \& Harris, J. A. (2010). Latent inhibition. In C. J. Mitchell \& M. E. Le Pelley (Eds.), Attention and associative learning: From brain to behaviour (pp. 99-132). Oxford, UK: Oxford University Press.

Ionescu, T. (2012). Exploring the nature of cognitive flexibility. New Ideas in Psychology, 30(2), 190-200. doi:10.1016/j.newideapsych.2011.11.001

Kamin, L. J. (1969). Predictability, surprise, attention, and conditioning. In B. A. Campbell \& R. M. Church (Eds.), Punishment and Aversive Behaviour (pp. 279-296). New York, NY: Appleton Century Crofts.

Kasof, J. (1997). Creativity and breadth of attention. Creativity Research Journal, 10(4), 303-315. doi:10.1207/s15326934crj1004_2 
Kéri, S. (2011). Solitary minds and social capital: Latent inhibition, general intellectual functions and social network size predict creative achievements. Psychology of Aesthetics, Creativity, and the Arts, 5(3), 215-221. doi:10.1037/a0022000

Le Pelley, M. E., Mitchell, C. J., Beesley, T., George, D. N., \& Wills, A. J. (2016). Attention and associative learning in humans: An integrative review. Psychological Bulletin, 142(10), 1111-1140. doi:10.1037/bul0000064

Le Pelley, M. E., \& Mclaren, I. P. L. (2001). The mechanics of associative change. In Proceedings of the 27th Annual Meeting of the Cognitive Science Society (pp. 534-539). Mahwah, NJ: Lawrence Erlbaum Associates.

Le Pelley, M. E., \& McLaren, I. P. L. (2003). Learned associability and associative change in human causal learning. The Quarterly Journal of Experimental Psychology Series B, Comparative and Physiological Psychology, 56(1), 68-79.

Le Pelley, M. E., \& McLaren, I. P. L. (2004). Associative history affects the associative change undergone by both presented and absent cues in human causal learning. Journal of Experimental Psychology: Animal Behavior Learning and Cognition, 30(1), 67-73. doi:10.1037/0097-7403.30.1.67

Le Pelley, M. E., Vadillo, M., \& Luque, D. (2013). Learned predictiveness influences rapid attentional capture: Evidence from the dot probe task. Journal of Experimental Psychology. Learning, Memory, and Cognition, 39(6), 1888-1900. doi:10.1037/a0033700

Lewis, T. (2009). Creativity in technology education: Providing children with glimpses of their inventive potential. International Journal of Technology and Design Education, 19(3), 255-268. doi:10.1007/s10798-008-9051-y

Lochmann, T., \& Wills, A. (2003). Predictive history in an allergy prediction task. In Schmalhofer, F., Young, R. M., \& Katz, G. (Eds.). Proceedings of EuroCogSci 03: The European Cognitive Science Conference (pp. 217-222). Retrieved from http://elliewills.co.uk/pubs/2003lochmannwills.pdf

Lubow, R. E., Alek, M., \& Arzy, J. (1975). Behavioral decrement following stimulus preexposure: Effects of number of preexposures, presence of a second stimulus, and interstimulus interval in children and adults. Journal of Experimental Psychology: Animal Behavior Processes, 104(2), 178-188. doi:10.1037/0097-7403.1.2.178

Lubow, R. E., \& Moore, A. U. (1959). Latent inhibition: The effect of nonreinforced preexposure to the conditional stimulus. Journal of Comparative and Physiological Psychology, 52(4), 415-419. doi:10.1037/h0046700

Lubow, R. E., Schnur, P., \& Rifkin, B. (1976). Latent inhibition and conditioned attention theory. Journal of Experimental Psychology: Animal Behavior Learning and Cognition, 2(2), 163-174. doi:10.1037/0097-7403.2.2.163

Mackintosh, N. J. (1975). A theory of attention: Variations in the associability of stimuli with reinforcement. Psychological Review, 82(4), 276-298. doi:10.1037/h0076778

Mackintosh, N. J., \& Little, L. (1969). Intradimensional and extradimensional shift learning by pigeons. Psychonomic Science, 14(1), 5-6. doi:10.3758/BF03336395 
Mendelsohn, G., \& Griswold, B. (1966). Assessed creative potential, vocabulary level, and sex as predictom of the use of incidental cues in verbal probtem solving. Journal of Personality and Social Psychology, 4, 423-431.

Meyersburg, C. A., Carson, S. H., Mathis, M. B., \& McNally, R. J. (2014). Creative histories: Memories of past lives and measures of creativity. Psychology of Consciousness: Theory, Research, and Practice, 1(1), 70-81. doi:10.1037/css0000004

Pearce, J. M., \& Hall, G. (1980). A model for Pavlovian learning: Variations in the effectiveness of conditioned but not of unconditioned stimuli. Psychological Review, 87(6), 532-552. doi:10.1037/0033-295X.87.6.532

Peterson, J. B., Smith, K. W., \& Carson, S. (2002). Openness and extraversion are associated with reduced latent inhibition: Replication and commentary. Personality and Individual Differences, 33(7), 1137-1147. doi:10.1016/S0191-8869(02)00004-1

Plucker, J. A., Beghetto, R. A., \& Dow, G. T. (2004). Why isn't creativity more important to educational psychologists? Potentials, pitfalls, and future directions in creativity research. Educational Psychologist, 39(2), 83-96. doi:10.1207/s15326985ep3902_1

Runco, M. A., Walczyk, J. J., Acar, S., Cowger, E. L., Simundson, M., \& Tripp, S. (2014). The incremental validity of a short form of the ideational behavior scale and usefulness of distractor, contraindicative, and lie scales. The Journal of Creative Behavior, 48(3), 185-197. doi:10.1002/jocb.47

Sawyer, R. K. (2012). Explaining creativity: The science of human innovation (2 ${ }^{\text {nd }}$ ed.). Oxford, UK: Oxford University Press.

Shepp, B. E., \& Eimas, P. D. (1964). Intradimensional and extradimensional shifts in the rat. Journal of Comparative and Physiological Psychology, 57(3), 357-361. doi:10.1037/h0043967

Wills, A. J., Lavric, A., Croft, G. S., \& Hodgson, T. L. (2007). Predictive learning, prediction errors, and attention: evidence from event-related potentials and eye tracking. Journal of Cognitive Neuroscience, 19(5), 843-854. doi:10.1162/jocn.2007.19.5.843

Zabelina, D. L., \& Robinson, M. D. (2010). Creativity as flexible cognitive control. Psychology of Aesthetics, Creativity, and the Arts, 4(3), 136-143. doi:10.1037/a0017379 